\title{
Chapter 18 \\ Cancer Management at Sites with Limited Resources: Challenges and Potential Solutions
}

\author{
Shahrukh K. Hashmi, Fady Geara, Asem Mansour, and Mahmoud Aljurf
}

The World Bank defines low-income economies ( $\$ 1005$ or less GNI per capita) or lower middle-income economies (\$1006 to \$3955 GNI per capita) as Low- and Middle-Income Countries (LMIC) which comprise of approximately two-thirds of the world's 197 countries recognized by the United Nations.

In the LMICs, population pyramids are changing dynamically towards a constrictive pattern from an expansive pattern as the lifetime expectancy is increasing due to an ageing population [1]. As age is the greatest risk factor for cancers [2, 3], expectedly the incidence of cancer continues to increase dramatically in LMIC, which is also partly due to advancements in diagnostic techniques [4]. The majority of cancer cases globally now occur in LMIC, and $65 \%$ of cancer deaths worldwide occur in these countries [5].

Apart from economic factors, there are considerable differences in the geopolitical arenas of LMIC compared to many developed nations [6, 7]; therefore the

\section{Authorship Contributions:}

SKH wrote the first draft of the manuscript. All authors vouch for the accuracy and contents of the manuscript. All authors approved the final version of the draft

\footnotetext{
S. K. Hashmi $(\bowtie)$

Division of Hematology, Department of Medicine, Mayo Clinic, Rochester, MN, USA

Sheikh Shakhbout Medical City, Abu Dhabi, UAE

e-mail: hashmi.shahrukh@mayo.edu

F. Geara

Naef K. Basile Cancer Institute, Department of Radiation Oncology, American University of Beirut, Beirut, Lebanon
}

\author{
A. Mansour \\ King Hussein Cancer Center, Amman, Jordan
}

M. Aljurf

Adult Hematology and HSCT, Oncology Center, King Faisal Specialist Hospital and

Research Center, Riyadh, Saudi Arabia 
challenges of providing comprehensive cancer care in the LMIC are different. Here, we will briefly summarize the current challenges that many LMICs face with respect to oncologic care, focusing on both prevention and therapeutics, with the caveat that these are general issues which may not apply to all LMICs.

\section{Medical Services, Data, and Infrastructure}

\section{Need for Effective cancer Registries}

A database or a registry of the cancer cases encompassing the type of tumor, stage, genomics, and other parameters is essential for overall cancer care for any country. This database could be a hospital-based or a population-based registry. Concept of population-based cancer registries is at least half a century old, as in 1950s, the American College of Surgeons (ACoS) implemented policies for development of hospital-based cancer registries. Sweden was the first country globally to establish a formal cancer registry for all cases diagnosed and linked the data with personal information (Swedish PIN and other variables). In 1973, The National Cancer Institute of the United States (US), through its Surveillance, Epidemiology and End Results (SEER) program established the first national cancer registry program which currently produces a variety of data (and analytics) on various aspects of cancer epidemiology [8].

Some LMICs have established cancer registries at federal level; however, the quality control mechanisms of these registries are extremely variable, and quite often data is either missing, or lags current data entry mechanisms which leads to consider delays in updating the data. Moreover, lack of long-term outcomes data would preclude accurate mortality or morbidity analysis. The need of accurate data of cancer cases in a country is imperative to develop cancer control programs and screening guidelines. Unless the burden of each cancer type and subtype is known, it is hard to allocate appropriate resources for prevention or treatment of the specific types of cancer. Additionally, there are a number of international grants or programs specifically for cancer-associated activities in LMICs; however, one needs to know the exact burden of the disease in order to apply for most of these grants. Therefore, the need for a cancer registry at national level should be a top priority of a country's healthcare policy. A state-of-the-art cancer center's hospital-based registry is insufficient to formulate future planning for cancer control, since it is limited to the patients coming to that center, and also due to the known phenomenon of patient shopping at various institutions (and second opinions), which may be more pronounced in the LMICs.

Given the importance of a nationwide cancer registry covering both urban and rural areas, as a solution, we would encourage both the governmental agencies and the institutional leadership to work together to develop policies for a mandatory cancer reporting mechanism and a national registry that would capture important 
variables (including outcomes data) and have resources to functionally sustain the registry in long run (technologic tools, data managers, statisticians etc.). The World Health Assembly in conjunction with World Health Organization (WHO) has passed the resolution Cancer Prevention and Control and has urged the governments to accelerate action to achieve the targets specified in the Global Action Plan and the "2030 United Nations Agenda for Sustainable Development". Establishment of effective cancer registries is an integral part of the proposed agenda by WHO.

Moreover, The World Bank's Regional Program of Cancer Registries (P163187) has proposed help in establishing population-based cancer registries to collect, analyze, and publish a regional compilation of cancer statistics. This is another avenue which can be evaluated for establishment of cancer registry at regional level.

\section{Lack of Connectivity}

Quite often, patients care is fragmented across the institutions. Some patients would receive initial consult at one institution, then laboratory work at another, and then radiology at a third institution. Not only the care is fragmented which can result in delay in both diagnosis and treatment, but also the information technology (IT) systems are not interfaced with each other thereby care is further affected. Having a unified IT system, or electronic medical record (EMR) systems that can interface with each other would make care better, and also would allow for a smooth transition of care between institutions. Another layer of approvals required for sharing information is the lack of clear cybersecurity laws for exchanging EMRs. Thus a conscious effort has to be made to clarify pathways for information exchange between institutions so that patient care is not affected.

\section{Research Infrastructure}

The improvement in the cancer survival is mainly due to tremendous investment in preclinical and clinical research by institutions (e.g., tertiary care hospitals), governmental agencies (e.g., National Institutes of Health), and the pharmaceutical industry. However, most of the innovation and discovery in oncology (including genomics and drug development) has come from investigators from the developed countries. A robust research infrastructure is required to assimilate all three phases of research, that is, basic science, translational, and clinical trials.

Basic science: The investment in preclinical research requires considerable resources that include laboratory and equipment as well as expertise [9]. Scientific collaborations with global experts is necessary at least in initial phases of setup of basic science laboratories, which require expensive equipment (and reagents) and human resources ( $\mathrm{PhD}$, and advanced technicians, besides postdoctoral trainees). 
Clinical trials: For clinical research, the institutional leadership and the governmental agencies must invest both in infrastructure (e.g., clinical trials unit) and in human resources [10]. The phase I, II, and III clinical trials require funding as expertise, and once the infrastructure is available (including CRCs, clinical trialists, and biostatisticians), then physician-scientists would be able to produce (write up protocols and successfully accrue the projected number of subjects) results from clinical research, and moreover attract cutting-edge clinical trials from pharmaceutical and biotech industry. Typically, randomized trials (mostly phase III) change the clinical practice, and over the past few decades, successful completion of randomized trials initiated by principal investigators from developing countries has been extremely rare $[11,12]$. Most common pathway of executing clinical trials in LMICs is facilitation by pharmaceutical industry, and occurrence of phase I clinical trials is hardly existent in most of the LMICs [13]. Having physician-scientists who are appreciated and given ample time and resources for research is a necessary factor for the successful execution of a clinical trial.

Translational research: Some LMICs have advanced facilities for basic science research and excellent tertiary care hospitals as well both producing some degree of scientific output. To translate the preclinical models into medical field requires direct collaboration between institutions. Unless this happens, cutting-edge innovation may not occur.

A comprehensive research infrastructure would require (apart from HR, space, and equipment), effective Institutional Review Board (IRB), Data Safety Monitoring Board (DSMB), and extensive collaborations with the regulatory agencies (e.g., a country's federal drug authority or equivalent agency). How to operationalize such an infrastructure requires a huge setup which has four essential aspects - human resources (scientists, physicians, nurses, clinical research coordinators, biostatistician, clinical nurses, pharmacists, phlebotomists) physical space (including negative pressure and positive pressure rooms), equipment (laboratory and office), and software (REDCap or other databases). This requires direct interaction with local health authorities.

\section{Tumor Boards and Multispecialty Care}

Recent data has indicated that multidisciplinary care and decisions improve outcomes of cancer patients [14]. A multidisciplinary tumor board may require expertise from radiologists, medical oncologists, radiation oncologists, surgeons, pathologists, and other specialties. This pertains more to solid cancers; however, in hematologic malignancies, molecular hematology boards have become routine to discuss the cases and diagnostic dilemmas at large cancer centers in developed countries. Emphasis should be laid in assimilation of such tumor boards for cancer subtypes in developing countries, which can also foster holistic care to a patient apart from increasing research collaborations between various clinical specialties. If 
multispecialty tumor boards cannot be established due to limited resources or lack of expertise, then efforts could be concentrated on scheduling regular tumor board meetings at a partner institution with a large cancer center (maybe within the same country or internationally).

Building a homogeneous multidisciplinary tumor board can indeed be very challenging at the beginning. Apart from utilizing technology to have virtual boards, one must continue to organize a team to eventually establish functional tumor boards this would require at least a medical oncologist, radiation oncologist, surgical oncologist, radiologist, pathologist, oncology nurses, coordinators, and ideally also palliative care team as well.

\section{Human Resources}

\section{Unavailability of Specialized, but Essential Cancer Services and Human Power Radiotherapy and Stem Cell Transplantation}

There are many services that are necessary for both curative and palliative management in cancers; however, due to expertise required, technology transfer issues, equipment infrastructure, and costs, they may be unavailable in LMICs. On the top of these essential services are stem cell transplantation (SCT) $[15,16]$ and radiotherapy [17-19]. These two services are necessary for many cancers and may be the only potential cure, and in order to achieve this cure, many patients from LMIC may travel outside their home country for the receipt of these services. One essential element of these services is that efforts must be concentrated to start with bare minimum requirements in order to establish at least one specialized unit, for example, for radiation therapy unit in a country, it is not essential to wait for funding and expertise for carbon-ion therapy or proton beam therapy, but can start with the cobalt therapy or with traditional linear accelerators. Similarly, for SCT, it is not imperative to have a huge infrastructure for allogeneic SCTs, when a center can start with the relatively low-risk autologous SCTs. Apart from getting expertise from outside the country for a new specialized program, one has to consider capacity building and skills training for physicians and nurses within a country.

\section{Education Infrastructure}

Efforts to train physicians, nurses, and technicians for specialized care is essential. This applies directly to the fields of cancer in general, but more so to the very specialized fields, for example, radiation oncology and bone marrow transplantation. There should be concentrated effort by the institutional leadership and also by the governmental agencies who regulate the medical training programs. 


\section{Quality Management and Access to Care}

\section{Sustainability and Consistency}

Sustainability and consistency in quality of cancer care is a challenge in unpredictable geopolitical circumstances and, therefore, a great degree of disparity exists in the quality level among various providers (and/or institutions). Given there is a lack of effective reporting and measurement of outcomes, we propose to have simplified metrics for objective evaluation of quality of care so that the results can be shared to identify gaps and acquire best practices.

\section{Access to cancer Care}

In many countries, access to cancer care is extremely hard due to many factors particularly due to the shortage of comprehensive services, lack of expertise, and due to economic hardships due to a lack of comprehensive national coverage. Even if there is a well-established cancer center, populations may not have the resources to reach to the specialized centers due to social and financial barriers.

A study from Cameroon indicated a 6 month delay between appearance of the first sign of cancer and seeing a healthcare provider [20]. Access to a cancer specialist is significantly delayed due to a variety of reasons in LMIC, which include (but not limited to), cultural preferences (e.g., taboo in some communities) and trust issues with medical providers or allopathic physicians (with a greater emphasis on complementary and alternative medicine therapies), financial reasons, or due to a deficiency of oncologists. This must be addressed at both institutional level and national health policy level. The governmental structure should be able to cope up with the increasing demand for treating oncologists in LMICs. Moreover, disparities in access to cancer specialists and cancer-related services (e.g., PET scans, radiation oncology facility, etc.) should be addressed. Some methods could potentially be applied to improve access, for example, targeted fundraising and donations, support group initiation, negotiating with government sponsoring of cancer programs and services, and revenue sharing programs with industry.

\section{Influence of Political Activities: Refugee Crises and Internal Displacement}

According to the UN Refugee Agency (UNHCR), at least 79.5 million people around the world have been forced to flee their homes which includes nearly 26 million refugees and 47 million internally displaced people (IDP) by 2019 [21]. This leads to additional burdens for social and healthcare-related expenditures to the 
countries. Not only delayed diagnosis of the refugees and IDPs can lead to increased mortality, the psychosocial issues in these people can lead to considerably increased morbidity.

There has to be a concentrated effort by the governmental agencies to evaluate the exact oncologic needs of the refugees and IDPs. Mechanisms must be established to provide both urgent care and long-term planning if the refugees and IDPs are diagnosed with cancer or received initial treatment at their home country or local city before being displaced. Many hospitals may not accept refugees for cancer care, but it is the government's responsibility to provide at least emergency oncology care to the refugees and IDPs, and these include but are not limited to spinal cord compression, hypercalcemia of malignancy, superior-vena-cava syndrome, acute leukemias (ALL, APL, AML), and high-grade lymphomas (particularly Burkitt lymphoma and large cell lymphomas).

It is critical that the international or local governmental support provided by the stakeholders focus on noncommunicable diseases as hematologic and oncologic emergencies are almost always fatal if not treated urgently.

\section{Successful Models of Cancer Care: Sharing Best Practices}

Given a multitude of differences between LMIC and developed world with respect to cancer care, especially in regard to socioeconomic status (SES) of patients, regulatory agency policies, and political climates, a potential solution to successfully implement oncologic care so that outcomes in the end users improve is to partner with countries in which successful execution of the cancer programs has already occurred. This would ideally be a concept of twinning which has prevailed in developing countries to many projects both in healthcare and other sectors. A classic example is the twinning program for the establishment of a stem cell transplant program in Bangladesh with the help of expertise at every level (architecture/design, laboratory issues, transplant physician expertise, nursing training, and others), with a hospital in Boston, Massachusetts, which has resulted in successful example of technology transfer from a developed country to LMIC.

For sharing best practices, it is not essential to have physical presence of large teams in a LMIC institution or governmental agency, and a virtual contract for telehealth could also be of help for smaller projects, for example, for tumor boards.

Nonetheless, a unified standard approach is essential for a country's success so that most successful practices which have proven to improve clinical outcomes can be shared. In this instance, it is imperative that there is one professional organization which dictates not only national guidelines but also sets up research priorities. Although this seems trivial, however, in real-world practice, there are critical challenges with respect to establishing or sustaining professional organizations. For example, in some LMIC there may be two or even three professional hematology or oncology societies, all claiming national statuses in clinical and research matters. This practice of egoistic approach based on institutional or personal prides must 
end, and a unified organization representing the entire nation should be established. In the USA, for instance, there is one national organization for each subspecialty, for example, ASCO for medical oncology, ASTRO for radiation oncology, and ASH for hematology. To have some control to either mandate or at least encourage one national society will lead to less dispersion of knowledge and best practices.

An ideal model to cover cancer care in all LMICs does not exist; however, various models fit into the geopolitical and economic infrastructure of a country, for example, matrix cancer centers or stand-alone centers may fit into the management paradigm of different countries.

\section{Drug Approvals and Shortages}

Drug approval processes in the LMIC vary considerably and quite often is a constantly changing process with layers of bureaucratic and political interventions. Each LMIC, like the developed countries, has a drug regulatory authority or agency; however, efficiency differs tremendously and is influenced not only by intervening individuals with authority but also by pharmaceutical industry. Given immunotherapy especially with checkpoint inhibitors is being increasingly used for treatments of various cancers; its regulation and importation poses a constant challenge to developing countries. It is predicted that immunotherapies will replace majority of the cytotoxic chemotherapies in the near future for most of the cancers. Thereby, regulatory agencies of LMIC need to have dedicated staff for establishment of effective policies for rapid approval of essential cancer drugs.

Essential cancer drugs are often not available, not accessible, or not used appropriately in LMICs which is one of the greatest dilemmas of oncologic care [22]. Drug shortages are typically more common in LMICs and can lead to devastating outcomes in cancer patients [23]. This aspect of drug shortages is a critical, yet relatively neglected issue within the cancer management paradigm, and downstream the cost of cancer care can potentially increase tremendously. For example, if vincristine deficiency is sustained for few weeks or months, then it could result in a domino effect as this drug is the backbone of treatment of pediatric ALL, and if unavailable, can lead to multiple relapses, the treatment of which may be extremely complicated (including perhaps an allogeneic stem cell transplant) and expensive.

In 2011, the Council of International Pharmaceutical Federation called on "all stakeholders, including governments, pharmaceutical manufacturers, pharmacy wholesalers, pharmaceutical purchasing agencies, medicine insurance plans, pharmaceutical regulators and the pharmacy profession to urgently evaluate these issues and work to ensure continuity of medication supply so that the appropriate treatment of patients can be initiated and maintained". One of the solutions is to ensure smooth network of supply chain between the institutions that carry cancer drugs so that transfer can be ensued where the greatest need is apparent. Moreover, technologic advancements including machine learning algorithms (described below) can 
augment a smooth drug supply chain by not only prompting exchanges but also providing predictions for shortage of essential medications.

Pricing models that apply to the North American and European countries do not apply to the LMICs. Models where cost-sharing or cost-containment policies that can be enacted should be sought, and if not possible, then innovative strategies that can derive delivery of medications to the end users must happen. In India and Brazil, for some chemotherapy drugs, the pharmaceutical industry and the government have negotiated contracts to ensure cheaper drug availability to the cancer patients (subsidized cost versus on a governmental plan), and this includes local manufacturing for certain specialized medications. This is not an easy task, and would need to conform to the international patency laws; however, it is achievable as experienced by the abovementioned LMICs. Applications of generic medications and biosimilars should increase once equivalence in safety and efficacy is established.

\section{Essential Need for a Safe and Effective Institutional Blood Bank}

In many developing countries, it can be challenging to secure matched platelets and packed red blood cells in a timely fashion. This issue is complicated by the fact that some federal authorities in some countries restrict the blood banking and donor procedures to a central blood bank supervised by the government officials, and thus distribution can be at risk. This issue must be tackled before establishing a comprehensive cancer center, as many patients undergoing chemotherapy or stem cell transplantation require massive amounts of transfusion.

\section{Applying Technologic Advancements in Oncology: Artificial Intelligence and Internet-of-Things}

The technologic advancements are revolutionizing the cancer care especially with respect to diagnostics particularly in pathology and radiology [24]. The availability of big data has provided ample opportunity to evaluate and analyze predictive models in hematology [25, 26], medical oncology [27-29], and radiation oncology [3032] via machine learning algorithms [33]. Apart from software development, the Internet-of-Things which runs on 5G network [34] is well posed to direct many aspects of healthcare including telehealth [35]. Some LMICs have already adapted these technology-based tools to improve the management of hematologic and oncologic management; though it requires initial investment in hardware/software technology and human resources, in long run, these technologies are predicted to lower cost of care and improve efficiencies. Thereby, we strongly propose to consider incorporation of technologic advancements in the current cancer framework going forward if possible and resources allow. 


\section{Unavailability of Specialized, but Essential Cancer Services: Radiotherapy and Stem Cell Transplantation}

There are many services that are necessary for both curative and palliative management in cancers; however, due to expertise required, technology transfer issues, equipment infrastructure, and costs, they may be unavailable in LMICs. On the top of these essential services are stem cell transplantation (SCT) $[15,16]$ and radiotherapy [17-19]. These two services are necessary for many cancers and may be the only potential cure, and in order to achieve this cure, many patients from LMIC may travel outside their home country for the receipt of these services. One essential element of these services is that efforts must be concentrated to start with bare minimum requirements in order to establish at least one specialized unit, for example, for radiation therapy unit in a country, it is not essential to wait for funding and expertise for carbon-ion therapy or proton beam therapy, but can start with the cobalt therapy or with traditional linear accelerators. Similarly, for SCT, it is not imperative to have a huge infrastructure for allogeneic SCTs, when a center can start with the relatively low-risk autologous SCTs. Apart from getting expertise from outside the country for a new specialized program, one has to consider capacity building and skills training for physicians and nurses within a country.

Above, we mention some of the challenges and propose potential solutions for these issues pertaining to LMICs with respect to cancer care. It is apparent that onesize-fits-all solution is impractical and an approach tailored towards individualizing the priorities within a country given its resources is the most practical way of successfully implementing comprehensive cancer care.

\section{Public Health Crises}

\section{Carcinogen Prevention}

The tobacco epidemic due to a high prevalence of smoking has led to a much higher incidence of smoking-related cancers in the developing countries [36, 37]. While in the United States, the prevalence of smoking has decreased over the past few decades, in the developing countries, this trend has not been observed, and therefore, it is very likely that for the next few years there is a predictable increase in the incidence rates of cancers. Apart from cigarette smoking, certain behavioral practices are much more prevalent in the developing countries that also contribute to the increase in certain cancers in these populations which include betel nut (especially when mixed with slate lime) [38], herbal cigarettes [39], and shisha (pot smoking) [40]. 


\section{Late Diagnosis}

Due to either a lack of public health mandate for screening or non-implementation of policies of the screening programs, majority of the cancers are seen at a laterstage diagnosis.

Significant investment in the infrastructure for the above is needed. In the current era of media, the message also needs to get across to the public as well, and the media tools, for example, WhatsApp, Twitter, television, and direct-to-consumer messages could be tremendously helpful. Moreover, getting support by international organizations that have established programs for prevention and management of cancers should ideally be undertaken. Union for International Cancer Control (UICC) is one such nongovernmental organization that opens to membership to all developing and developed countries.

\section{Conclusions}

Above, we mention some of the challenges and propose potential solutions for these issues pertaining to LMICs with respect to cancer care. Some of the solutions are covered after each issue mentioned above. Some umbrella solutions include seeking funding by collaboration with charity and philanthropic organizations for financial and also in-kind support (patient transportation, housing, food, etc.). Holding fundraising events in collaboration with other government or private agencies could also be tremendously helpful.

It is apparent that one-size-fits-all solution is impractical and an approach tailored towards individualizing the priorities within a country given its resources is the most practical way of successfully implementing comprehensive cancer care.

Conflicts of Interest None of the authors declare any relevant COI.

Disclosures: SKH has received funding from Mallinckrodt, Pfizer, Novartis, Janssen.

SKH has received travel grants from MSD, Takeda, Gilead, and BMS.

\section{References}

1. Roser M, Ritchie H, Ortiz-Ospina E. World population growth. Our World in Data; 2013.

2. Kennedy BK, Berger SL, Brunet A, Campisi J, Cuervo AM, Epel ES, Franceschi C, Lithgow GJ, Morimoto RI, Pessin JE, Rando TA. Geroscience: linking aging to chronic disease. Cell. 2014;159(4):709-13.

3. Niccoli T, Partridge L. Ageing as a risk factor for disease. Curr Biol. 2012;22(17):R741-52.

4. Bellanger M, Zeinomar N, Tehranifar P, Terry MB. Are global breast cancer incidence and mortality patterns related to country-specific economic development and prevention strategies? J Global Oncol. 2018;4:1-16. 
5. Torre LA, Bray F, Siegel RL, Ferlay J, Lortet-Tieulent J, Jemal A. Global cancer statistics, 2012. CA Cancer J Clin. 2015;65(2):87-108.

6. De Souza JA, Hunt B, Asirwa FC, Adebamowo C, Lopes G. Global health equity: cancer care outcome disparities in high-, middle-, and low-income countries. J Clin Oncol. 2016;34(1):6.

7. Navarro V, Muntaner C, Borrell C, Benach J, Quiroga Á, Rodríguez-Sanz M, Vergés N, Pasarín MI. Politics and health outcomes. Lancet. 2006;368(9540):1033-7.

8. https://seer.cancer.gov/about/. Accessed 07-07-2020.

9. Rochmyaningsih D. The developing world needs basic research too. Nature. 2016;534(7605):7-7.

10. Bosnjak Pasic M, Vidrih B, Sarac H, Pasic H, Vujevic L, Soldo Koruga A, Rajic F. Clinical trials in developing countries-ethical considerations. Psychiatr Danub. 2018;30(3):285-91.

11. Arabi YM, Al-Hameed F, Burns KE, Mehta S, Alsolamy SJ, Alshahrani MS, Mandourah Y, Almekhlafi GA, Almaani M, Al Bshabshe A, Finfer S. Adjunctive intermittent pneumatic compression for venous thromboprophylaxis. N Engl J Med. 2019;380(14):1305-15.

12. Jehan F, Nisar I, Kerai S, Balouch B, Brown N, Rahman N, Rizvi A, Shafiq Y, Zaidi AK. Randomized trial of amoxicillin for pneumonia in Pakistan. $N$ Engl J Med. 2020;383(1):24-34.

13. Odedina FT, Shamley D, Okoye I, Ezeani A, Ndlovu N, Dei-Adomakoh Y, Meza K, Agaba R, Fathi P, Askins N. Landscape of oncology clinical trials in Africa. JCO Global Oncol. 2020;6:932-41.

14. Specchia ML, Frisicale EM, Carini E, Di Pilla A, Cappa D, Barbara A, Ricciardi W, Damiani G. The impact of tumor board on cancer care: evidence from an umbrella review. BMC Health Serv Res. 2020;20(1):73.

15. Hashmi SK, Srivastava A, Rasheed W, Adil S, Wu T, Jagasia M, Nassar A, Hwang WY, Hamidieh AA, Greinix HT, Pasquini MC. Cost and quality issues in establishing hematopoietic cell transplant program in developing countries. Hematol Oncol Stem Cell Ther. 2017;10(4):167-72.

16. Aljurf M, Weisdorf D, Hashmi SK, Nassar A, Gluckman E, Mohty M, Rizzo D, Pasquini M, Hamadani M, Saber W, Hari P. Worldwide Network for Blood and Marrow Transplantation (WBMT) recommendations for establishing a hematopoietic stem cell transplantation program in countries with limited resources (Part II): clinical, technical and socio-economic considerations. Hematol Oncol Stem Cell Ther. 2020;13(1):7-16.

17. Parkes J, Hess C, Burger H, AnacakY, Ahern V, Howard SC, Elhassan M, et al. Recommendations for the treatment of children with radiotherapy in low-and middle-income countries (LMIC): a position paper from the Pediatric Radiation Oncology Society (PROS-LMIC) and Pediatric Oncology in Developing Countries (PODC) working groups of the International Society of Pediatric Oncology (SIOP). Pediatr Blood Cancer. 2017;64:e26903.

18. Datta NR, Samiei M, Bodis S. Radiation therapy infrastructure and human resources in lowand middle-income countries: present status and projections for 2020. Int J Radiat Oncol Biol Phys. 2014;89(3):448-57.

19. Abdel-Wahab M, Bourque JM, Pynda Y, Iżewska J, Van der Merwe D, Zubizarreta E, Rosenblatt E. Status of radiotherapy resources in Africa: an International Atomic Energy Agency analysis. Lancet Oncol. 2013;14(4):e168-75.

20. Price AJ, Ndom P, Atenguena E, Mambou Nouemssi JP, Ryder RW. Cancer care challenges in developing countries. Cancer. 2012;118(14):3627-35.

21. https://www.unhcr.org/figures-at-a-glance.html. Accessed 07-09-2020.

22. https://www.who.int/bulletin/volumes/85/4/06-033647/en/. Accessed July 10th, 2020.

23. Acosta A, Vanegas EP, Rovira J, Godman B, Bochenek T. Medicine shortages: gaps between countries and global perspectives. Front Pharmacol. 2019;10:763.

24. Savage N. How AI is improving cancer diagnostics. Nature. 2020;579(7800):S14.

25. Lee SI, Celik S, Logsdon BA, Lundberg SM, Martins TJ, Oehler VG, Estey EH, Miller CP, Chien S, Dai J, Saxena A. A machine learning approach to integrate big data for precision medicine in acute myeloid leukemia. Nat Commun. 2018;9(1):1-13.

26. Salah HT, Muhsen IN, Salama ME, Owaidah T, Hashmi SK. Machine learning applications in the diagnosis of leukemia: current trends and future directions. Int J Lab Hematol. 2019;41(6):717-25. 
27. Yue W, Wang Z, Chen H, Payne A, Liu X. Machine learning with applications in breast cancer diagnosis and prognosis. Designs. 2018;2(2):13.

28. Wang H, Zhou Z, Li Y, Chen Z, Lu P, Wang W, Liu W, Yu L. Comparison of machine learning methods for classifying mediastinal lymph node metastasis of non-small cell lung cancer from 18 F-FDG PET/CT images. EJNMMI Res. 2017;7(1):11.

29. Manogaran G, Vijayakumar V, Varatharajan R, Kumar PM, Sundarasekar R, Hsu CH. Machine learning based big data processing framework for cancer diagnosis using hidden Markov model and GM clustering. Wirel Pers Commun. 2018;102(3):2099-116.

30. Shafai-Erfani G, Wang T, Lei Y, Tian S, Patel P, Jani AB, Curran WJ, Liu T, Yang X. Dose evaluation of MRI-based synthetic CT generated using a machine learning method for prostate cancer radiotherapy. Med Dosim. 2019;44(4):e64-70.

31. Giraud P, Giraud P, Gasnier A, El Ayachy R, Kreps S, Foy JP, Durdux C, Huguet F, Burgun A, Bibault JE. Radiomics and machine learning for radiotherapy in head and neck cancers. Front Oncol. 2019;9:174.

32. Valdes G, Solberg TD, Heskel M, Ungar L, Simone CB II. Using machine learning to predict radiation pneumonitis in patients with stage I non-small cell lung cancer treated with stereotactic body radiation therapy. Phys Med Biol. 2016;61(16):6105.

33. Chen B, Garmire L, Calvisi DF, Chua MS, Kelley RK, Chen X. Harnessing big 'omics' data and AI for drug discovery in hepatocellular carcinoma. Nat Rev Gastroenterol Hepatol. 2020;17:1-14.

34. Cheong IY, An SY, Cha WC, Rha MY, Kim ST, Chang DK, Hwang JH. Efficacy of mobile health care application and wearable device in improvement of physical performance in colorectal cancer patients undergoing chemotherapy. Clin Colorectal Cancer. 2018;17(2):e353-62.

35. Ray PP, Dash D, De D. A systematic review of wearable systems for cancer detection: current state and challenges. J Med Syst. 2017;41(11):180.

36. Islami F, Torre LA, Jemal A. Global trends of lung cancer mortality and smoking prevalence. Translat Lung Cancer Res. 2015;4(4):327.

37. Barta JA, et al. Global Epidemiology of Lung Cancer. Ann Glob Health. 2019;85(1):8, 1-16. https://doi.org/10.5334/aogh.2419.

38. https://www.who.int/mediacentre/news/releases/2003/priarc/en/\#: : text=A\% 20 CANCER $\% 2$ DCAUSING $\% 20$ HABIT\&text=Areca $\% 20$ nut $\% 2$ C $\% 20 \mathrm{a} \% 20 \mathrm{common} \% 20 \mathrm{com}$ ponent $\% 20$ of $\% 20$ all $\% 20$ betel $\% 20$ quid $\% 20$ preparations, itself $\% 20$ is $\% 20$ carcinogenic $\% 20$ to $\% 20$ humans.

39. Gan Q, Yang J, Yang G, Goniewicz M, Benowitz NL, Glantz SA. Chinese "herbal" cigarettes are as carcinogenic and addictive as regular cigarettes. Cancer Epidemiol Prevent Biomark. 2009;18(12):3497-501.

40. Maziak W. The waterpipe: an emerging global risk for cancer. Cancer Epidemiol. 2013;37(1):1-4.

Open Access This chapter is licensed under the terms of the Creative Commons Attribution 4.0 International License (http://creativecommons.org/licenses/by/4.0/), which permits use, sharing, adaptation, distribution and reproduction in any medium or format, as long as you give appropriate credit to the original author(s) and the source, provide a link to the Creative Commons license and indicate if changes were made.

The images or other third party material in this chapter are included in the chapter's Creative Commons license, unless indicated otherwise in a credit line to the material. If material is not included in the chapter's Creative Commons license and your intended use is not permitted by statutory regulation or exceeds the permitted use, you will need to obtain permission directly from the copyright holder.

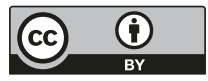

